\title{
Pseudorandom Functions and Permutations Provably Secure against Related-Key Attacks
}

\author{
Mihir Bellare and David Cash \\ Dept. of Computer Science \& Engineering, University of California San Diego, USA \\ $\{$ mihir, cdcash\}@cs.ucsd.edu \\ http://www.cs.ucsd.edu/users/\{mihir, cdcash
}

\begin{abstract}
This paper fills an important foundational gap with the first proofs, under standard assumptions and in the standard model, of the existence of PRFs and PRPs resisting rich and relevant forms of relatedkey attack (RKA). An RKA allows the adversary to query the function not only under the target key but under other keys derived from it in adversary-specified ways. Based on the Naor-Reingold PRF we obtain an RKA-PRF whose keyspace is a group and that is proven, under DDH, to resist attacks in which the key may be operated on by arbitrary adversary-specified group elements. Our framework yields other RKA-PRFs including a DLIN-based one derived from the LewkoWaters PRF. We show how to turn these PRFs into PRPs (blockciphers) while retaining security against RKAs. Over the last 17 years cryptanalysts and blockcipher designers have routinely and consistenly targeted RKA-security; it is important for abuse-resistant cryptography; and it helps protect against fault-injection sidechannel attacks. Yet ours are the first significant proofs of existence of secure constructs. We warn that our constructs are proofs-of-concept in the foundational style and not practical.
\end{abstract}

\section{Introduction}

Alarmed by the number of successful related-key attacks (RKAs) against real

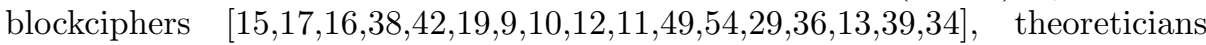
have stepped back to ask to what extent the underlying goal of RKA-secure PRFs and PRPs is achievable at all. The question is made challenging by the unusual nature of the attack model which allows the adversary to manipulate the key. Previous works providing RKA-secure PRFs and PRPs have bypassed rather than overcome the core technical difficulties by using the ideal cipher or random oracle models, making non-standard assumptions themselves "related-key" in nature, or limiting attackers to weak classes of RKAs for which the problem disappears [546]. We provide a new technical approach based on which we obtain the first designs of PRFs and PRPs secure against non-trivial and application-relevant forms of RKAs under standard assumptions (DDH) and in the standard model. Our constructions are not practical, providing, instead, in-principle proofs of achievability of the goals in the classical foundational style.

T. Rabin (Ed.): CRYPTO 2010, LNCS 6223, pp. 666-684, 2010.

(C) International Association for Cryptologic Research 2010 
THE MODEL. RKAs were introduced by Biham and Knudsen [7840] and formalized by Bellare and Kohno $(\mathrm{BK})[5]$. Referring to any $\phi: \mathcal{K} \rightarrow \mathcal{K}$ as a related-key deriving (RKD) function, the latter define what it means for a family of functions $F: \mathcal{K} \times \mathcal{D} \rightarrow \mathcal{R}$ to be a $\Phi$-RKA-PRF, where $\Phi$ is a class (set) of RKD functions. The game begins by picking a random challenge bit $b$, a random target key $K \in \mathcal{K}$ and, for each $L \in \mathcal{K}$, a random function $G_{L}: \mathcal{D} \rightarrow \mathcal{R}$. The adversary is allowed multiple queries to an oracle that, given a pair $(\phi, x) \in \Phi \times \mathcal{D}$, returns $F_{\phi(K)}(x)$ if $b=1$ and $G_{\phi(K)}(x)$ if $b=0$, and its advantage is $2 \operatorname{Pr}\left[b=b^{\prime}\right]-1$, where $b^{\prime}$ is the bit it outputs. The definition of a family of permutations (blockcipher) $F: \mathcal{K} \times \mathcal{D} \rightarrow \mathcal{D}$ being a $\Phi$-RKA-PRP is analogous, the difference being that each $G_{L}$ is a random permutation on $\mathcal{D}$ rather than a random function. Note that when $\Phi$ consists of just the identity function, we recover the standard PRF [33] and PRP [45] notions.

Group-INDUCED CLASSES. We must beware of inherent limitations. It is observed in [5] that some $\Phi$ are "impossible" in the sense that no $F$ can be a $\Phi$-RKA-PRF or a $\Phi$-RKA-PRP. Indeed, any $\Phi$ that contains a constant function $\phi(\cdot)=C$, for some attacker-known constant $C \in \mathcal{K}$, is impossible. (For some $x$, just query the RK-oracle with $(\phi, x)$ and return 1 if the response is $F_{C}(x)$.) The class of all RKD functions is impossible, and so is the class of all permutations. The basic foundational question, then, is to identify specific classes $\Phi$, as rich, interesting and relevant as possible, for which we can prove "possibility," meaning existence of $\Phi$-RKA-PRFs and $\Phi$-RKA-PRPs. But which classes are good candidates?

BK [5] showed the (standard model) possibility of any class $\Phi$ whose member RKDs modify only the second half of the given key, and Lucks [46] gave, for the same class, an alternative construction with better concrete security. But if part of the key is unmodified, we can just use it as the "actual" key and put the rest in the input, meaning RKA-security here is for "trivial" reasons. For the proof-of-concept results in which we are interested, we seek candidate classes where the core technical difficulties cannot be bypassed in this way.

Luckily, Lucks [46] has already pinpointed a worthy target. His group-induced classes are elegant, appealing, non-trivial and application-relevant. If $(\mathcal{K}, *)$ is a group under an operation " $*$ ", the associated group-induced class is $\operatorname{rkd}[\mathcal{K}, *]=$ $\left\{\phi_{\Delta}^{*}: \Delta \in \mathcal{K}\right\}$ where $\phi_{\Delta}^{*}(K)=K * \Delta$ for all $K \in \mathcal{K}$. These classes are rich because all group actions are included. They also have what in [32] is called the completeness property and viewed as important to non-triviality of the class, namely that for any $K, K^{\prime} \in \mathcal{K}$ there is a $\phi \in \operatorname{rkd}[\mathcal{K}, *]$ such that $\phi(K)=$ $K^{\prime}$. Security relative to these classes suffices for applications and cannot be established by tricks such as the above. The quest that emerges is to find (nontrivial) groups $(\mathcal{K}, *)$ for which we can show the possibility of $\operatorname{rkd}[\mathcal{K}, *]$, meaning exhibit $\operatorname{rkd}[\mathcal{K}, *]$-RKA-PRFs and $\operatorname{rkd}[\mathcal{K}, *]$-RKA PRPs $F: \mathcal{K} \times \mathcal{D} \rightarrow \mathcal{R}$ whose keyspace is $\mathcal{K}$.

Previous WORK. Results of [5] imply that ideal ciphers achieve rkd[K, $*]-\mathrm{RKA}$ PRP security for any large enough group $(\mathcal{K}, *)$. Also, one can easily strengthen a given PRF or PRP to be a $r k d[\mathcal{K}, *]-\mathrm{RKA}$ one by hashing the key with a random 
oracle before use 46]. However, it is unclear how to instantiate the ideal primitives here to get "real" constructions for even a single group 2518]. For certain composite numbers $M$, Lucks [46] provides rkd $\left[\mathbb{Z}_{M},+\right]$-RKA-PRFs for the group $\left(\mathbb{Z}_{M},+\right)$, where + is addition modulo $M$, but the assumptions on which he bases security are not only interactive and novel but also themselves "related-key" in nature and uncomfortably close to just assuming the construct itself is secure, making the value of the proofs debatable from the point of view of security assurance. Existing PRFs such as the DDH-based one of Naor and Reingold [47] or the DLIN-based one of Lewko and Waters 43 are subject to simple attacks showing they provide no RKAsecurity. (Nonetheless they will be a starting point for our constructs.) Research has expanded to consider RKA-security of other primitives while leaving the goal unachieved for the more basic PRF, PRP and PRG ones 132.

The salient fact that emerges from this previous work is that we do not have even a single example of a group $(\mathcal{K}, *)$ for which we can prove the existence of a $\operatorname{rkd}[\mathcal{K}, *]-\mathrm{RKA}-\mathrm{PRF}$ or $\operatorname{rkd}[\mathcal{K}, *]$-RKA-PRP under standard assumptions in the standard model. The reason for the lack of progress is technical obstacles. The attack models underlying standard definitions of standard primitives do not allow any key-manipulation by the adversary. This makes it unclear how one can do any reductions, which seem to require applying RKD functions to an unknown key. This difficulty is appreciated, with Goldenberg and Liskov [32, Section 4] saying "The major open problem in related-secret security is whether or not related-key secure blockciphers exist ... related-secret pseudorandom bits cannot be constructed using traditional techniques. This leaves a significant open problem ... can fundamentally new techniques be found to create related-secret pseudorandom bits?"

NEW RKA-PRFs. We fill the above gap, providing the first constructions, under the standard DDH assumption and in the standard model, of $\Phi$-RKA-PRFs where $\Phi$ is group-induced. We obtain and analyze our designs via a general framework using two new primitives which may be of independent interest, namely key-malleable PRFs and key fingerprints. However, (surprisingly) at least one of our constructions, that we call the multiplicative DDH based RKA-PRF, is compact enough to state here. Let NR: $\left(\mathbb{Z}_{p}^{*}\right)^{n+1} \times\{0,1\}^{n} \rightarrow \mathbb{G}$ denote the Naor-Reingold PRF [47] that given key $\mathbf{a}=(\mathbf{a}[0], \ldots, \mathbf{a}[n]) \in\left(\mathbb{Z}_{p}^{*}\right)^{n+1}$ and input $x=x[1] \ldots x[n] \in\{0,1\}^{n}$ returns

$$
\mathrm{NR}(\mathbf{a}, x)=g^{\mathbf{a}[0] \prod_{i=1}^{n} \mathbf{a}[i]^{x[i]}},
$$

where $\mathbb{G}=\langle g\rangle$ is a group of prime order $p$. The keyspace $\mathcal{K}=\left(\mathbb{Z}_{p}^{*}\right)^{n+1}$ is a group under the operation $*$ of componentwise multiplication modulo $p$, but simple attacks [5] show that NR is not itself a $\operatorname{rkd}[\mathcal{K}, *]-\mathrm{RKA}-\mathrm{PRF}$. Let $h$ be a collisionresistant hash function with domain $\{0,1\}^{n} \times \mathbb{G}^{n+1}$ and range $\{0,1\}^{n-2}$. Given key a and input $x$, our construct $F:\left(\mathbb{Z}_{p}^{*}\right)^{n+1} \times\{0,1\}^{n} \rightarrow \mathbb{G}$ returns

$$
F(\mathbf{a}, x)=\operatorname{NR}\left(\mathbf{a}, 11 \| h\left(x,\left(g^{\mathbf{a}[0]}, g^{\mathbf{a}[0] \mathbf{a}[1]}, \ldots, g^{\mathbf{a}[0] \mathbf{a}[n]}\right)\right)\right),
$$

where "|l" denotes concatenation. Theorem 3 says that $F$ is a $\operatorname{rkd}\left[\left(\mathbb{Z}_{p}^{*}\right)^{n+1}, *\right]-$ RKA-PRF under the DDH assumption. The difficulty such a proof had to 
overcome was how the "simulator," given $\mathbf{d}$, can answer queries for $F$ on keys of the form $\mathbf{a} * \mathbf{d}$ without itself knowing a and without contradicting RKA security by enabling an attack.

This and other results are obtained via a general framework hinging on two new primitives. We call a $\operatorname{PRF} M: \mathcal{K} \times \mathcal{D} \rightarrow \mathcal{R}$ key-malleable relative to a class $\Phi$ of RKD functions on $\mathcal{K}$ if there is an efficient algorithm that given $(\phi, x) \in \Phi \times \mathcal{D}$ and oracle access to $M_{K}$ returns $M_{\phi(K)}(x)$. That this could be useful for building a $\Phi$-RKA-PRF is, on the one, hand, intuitive, because it allows us to simulate an oracle for $M(\phi(K), \cdot)$ via an oracle for $M(K, \cdot)$. But it is, on the other hand, counter-intuitive, because the same property immediately gives rise to an attack showing that $M$ is not a $\Phi$-RKA-PRF! Something else is necessary. This turns out to be the new concept of a key fingerprint, a vector $\mathbf{w}$ over $\mathcal{D}$ that uniquely identifies a key in the sense that for all $\left(\phi, \phi^{\prime}, K\right) \in \Phi \times \Phi \times \mathcal{K}$ we have $M_{\phi(K)}(\mathbf{w}) \neq M_{\phi^{\prime}(K)}(\mathbf{w})$ whenever $\phi \neq \phi^{\prime}$, where we have extended $M$ to vector second arguments on which it operates componentwise. Given $M, \mathbf{w}$ and a collision-resistant hash function, our general construction shows how to build $F: \mathcal{K} \times \mathcal{D} \rightarrow \mathcal{R}$ that we can show is a $\Phi$-RKA-PRF (cf. Theorem 1). The multiplicative DDH based RKA-PRF noted above is obtained by showing that $\mathrm{NR}$ is a key-malleable $\mathrm{PRF}$ relative to $\operatorname{rkd}\left[\left(\mathbb{Z}_{p}^{*}\right)^{n+1}, *\right]$ and then finding a key fingerprint for it. It is interesting that we turn malleability [28], typically viewed as a "bad" property, into a "good" property that we can exploit.

Two more constructs emanate from this framework. There are groups where DDH is easy but the Decision Linear (DLIN) problem of [22] still seems hard. Lewko and Waters 43] provide a DLIN-based analogue of the Naor-Reingold PRF, commenting that they know of no "closed-form" rendition of it akin to the above Equation (1) for NR. Using matrices, we provide in Equation (21) such a closed-form, and then, restricting attention to invertible matrices and slightly modifying the function, we obtain in Equation (22) a PRF that we can show is key-malleable and admits a key fingerprint. Our framework then yields a DLIN-based RKA-PRF [4].

The group $\left(\mathbb{Z}_{p}^{*}\right)^{n+1}$ underlying our multiplicative DDH-based RKA-PRF is, as the name indicates, multiplicative. Providing a DDH-based rkd $\left[\mathbb{Z}^{n+1}, *\right]-\mathrm{RKA}$ $\mathrm{PRF}$ where $*$ is componentwise addition modulo $p$ is more difficult. We provide in [4] a solution that involves first modifying the Naor-Reingold PRF and then applying our framework. However, the running time of our reduction is exponential in the input size. Theoretically, this means we must assume hardness of DDH against exponential-time algorithms. In practice, one can get security by using larger groups. This situation parallels that for the BB IBE scheme [20].

From RKA-PRFs To RKA-PRPs. Practical interest centers on RKA-secure blockciphers, meaning PRPs, and the constructions above are RKA-PRFs. It is not clear how one might modify the constructions to get RKA-PRPs. We use a different approach. Using deterministic extractors [2430 26], we convert our $\Phi$-RKA-PRFs into $\Phi$-RKA-PRGs with bitstring outputs. When these are used as key-derivation functions to key an ordinary (not RKA) PRP, we obtain a $\Phi$-RKA-PRP. (This second, composition step extends similar ones from [46]32]). 
For each class $\Phi$ for which we have a $\Phi$-RKA-PRF, this not only yields a CPAsecure $\Phi$-RKA-PRP but even a CCA-secure one.

Related WORK AND TECHniques. Based on the Boneh-Boyen short signature scheme 21, Dodis and Yampolskiy 27] define a PRF BBDY: $\mathbb{Z}_{p} \times S \rightarrow \mathbb{G}$ via $\operatorname{BBDY}(k, x)=\mathbf{e}(g, g)^{1 /(k+x)}$, where $\mathbf{e}:\langle g\rangle \times\langle g\rangle \rightarrow \mathbb{G}$ is a bilinear map and $S \subseteq \mathbb{Z}_{p}$. This had seemed to us promising towards building a rkd $\left[\mathbb{Z}_{p},+\right]$-RKAPRF, but (disappointingly) did not lead there. To begin with, BBDY is easily shown by attack to not itself be a rkd $\left[\mathbb{Z}_{p},+\right]$-RKA-PRF. (Adding 1 to $k$ or to $x$ yields the same outcome.) By exploiting the symmetry between $k$ and $x$ and using the composition paradigm, it turns out one can show how to construct a $\operatorname{rkd}\left[\mathbb{Z}_{p},+\right]-\mathrm{RKA}-\mathrm{PRF}$ if BBDY was a (plain) PRF, but only if the input domain $S$ was equal to $\mathbb{Z}_{p}$. The problem is that the q-DBDHI-based proof of 2721 requires $S$ to be "small" and in particular delivers nothing at all when $S=\mathbb{Z}_{p}$. We comment that there is no attack showing BBDY is not a PRF when $S=\mathbb{Z}_{p}$ and one might prove this in the generic model, but there seems little reason to pursue a generic group model solution when we already have a standard model, DDH-based solution. (In fact, since DDH is hard in the generic group model [53], our results already imply a generic model solution anyway.)

RKA-security is much easier for randomized primitives than deterministic ones. From the ElGamal scheme over a group of prime order $p$, one can easily get a (randomized) rkd $\left[\mathbb{Z}_{p},+\right]$-RKA-CPA-secure DDH-based symmetric encryption scheme. Applebaum [1] presents a more efficient rkd $\left[\{0,1\}^{n}, \oplus\right]$-RKA-CPAsecure (still randomized) symmetric encryption scheme assuming hardness of the LPN problem. There seems to be no simple way, from these techniques, to get the full-fledged group-induced RKA-PRFs that we target, where the computation is deterministic. That the deterministic case is more difficult than the randomized one is not surprising or unusual. In analogy, DDH based injective trapdoor functions [4] were discovered much later than DDH-based public-key encryption schemes.

Goldenberg and Liskov 32 broaden the scope to consider related-secret security. As with Lucks [46] they can, via composition, reduce the design of $\Phi$-RKAPRFs to the design of $\Phi$-RKA-PRGs, but provide no new constructions of the latter and hence of the former. They have negative results indicating the difficulty of getting these for non-trivial classes $\Phi$, and comment [32, Section 1] that "This leads us to the conclusion that if related-secret pseudorandomness (including related-key blockciphers) are possible, they must be proven either based on other related-secret pseudorandomness assumptions, or a dramatically new way of creating pseudorandomness from hardness must be developed." Our results are answers to these questions, showing that one can in fact obtain related-key pseudorandomness under standard assumptions. (Our RKA-PRFs of course directly yield RKA-PRGs.) Their negative results are in a limited model of computation and do not apply in our context.

Context. Conceived with the goal of studying the strength of blockcipher keyschedules 7840, RKAs quickly became mainstream. RKA-security is viewed as necessary for the collision-resistance of blockcipher-based compression 
functions [50]. (But one should note that this view has no formal justification.) RKA-resistance was a stated design goal of AES and remains so for other modern ciphers. A successful RKA is universally viewed by cryptanalysts as a break of the cipher. The recent attention-grabbing attacks on AES-192 and AES-256 17,16,15] were RKAs, and far from unique in this regard: a look at the literature shows that RKAs abound [3842 19 9|1012 114495429 36133934. Several higher-level cryptographic constructs, including HMAC 32 , the 3GPP confidentiality and integrity algorithms f8,f9 [35, and RMAC 3741, use related keys and thus rely for their (standard, not RKA) security on RKA-security of the underlying compression function or blockcipher.

The most direct use of RKA-security is for very cheap, simple and natural ways to rekey or tweak block ciphers. Subkeys of $K$ for use with modes of operation of a blockcipher $E$ might be derived in standard usage via $E_{K}\left(\Delta_{1}\right), E_{K}\left(\Delta_{2}\right), \ldots$ where $\Delta_{1}, \Delta_{2}, \ldots$ are constants. If $E$ is a RKA-PRP one can just use instead $K * \Delta_{1}, K * \Delta_{2}, \ldots$, where $*$ is a group operation, saving many blockcipher operations. On the other hand if $E$ is a $\operatorname{rkd}[\mathcal{K}, *]-\mathrm{RKA}-\mathrm{PRP}$, then $F_{K}^{T}(x)=$ $E_{K * T}(x)$ is shown in [5] to be a tweakable blockcipher, a primitive that has proven to be of great importance both conceptually and in applications [4451.

More designs would probably use related keys if it were possible to do so safely. Non-expert (in practice, most!) designers do it anyway, making RKA-security, in the words of Biryukov, Dunkelman, Keller, Khovratovich and Shamir [15], central to abuse-resistant cryptography.

Beyond this, RKA-security provides resistance to fault injection attacks 2314 where the attacker can inject faults that change bits of a hardware-stored key and observe the outputs of the cryptographic primitive under the modified key, putting RKAs under the umbrella of sidechannel attacks. This sidechannel connection is captured by the tamper-proof security model of Gennaro, Lysyanskaya, Malkin, Micali and Rabin [31. (They were apparently not aware of the prior model of [5] and the cryptanalytic literature on RKAs. We hope our current paper helps connect these two lines of work.)

Overall, the motivation for the theoretical study of RKA-security is not just powerful but unusual in coming from so many different parts of cryptography, namely foundations, cryptanalysis, protocol design and resistance to sidechannel attacks.

\section{Basic Definitions}

A family of functions $F: \mathcal{K} \times \mathcal{D} \rightarrow \mathcal{R}$ takes a key $K \in \mathcal{K}$ and input $x \in$ $\mathcal{D}$ and returns an output $F_{K}(x)=F(K, x) \in \mathcal{R}$. Let $\operatorname{FF}(\mathcal{K}, \mathcal{D}, \mathcal{R})$ be the set of all families of functions $F: \mathcal{K} \times \mathcal{D} \rightarrow \mathcal{R}$. For sets $X, Y$ let $\operatorname{Fun}(X, Y)$ be the set of all functions mapping $X$ to $Y$. If $S$ is a (finite) set then $s \stackrel{\$}{\leftarrow}$ denotes the operation of picking $s$ from $S$ at random and $|S|$ is the size of $S$. We denote by $y \stackrel{\$}{\leftarrow} A\left(x_{1}, x_{2}, \ldots\right)$ the operation of running randomized algorithm $A$ on inputs $x_{1}, x_{2}, \ldots$ and fresh coins and letting $y$ denote the output. If $\mathbf{v}$ is 
a vector then $|\mathbf{v}|$ denotes the number of its coordinates and $\mathbf{v}[i]$ denotes its $i$ th coordinate, meaning $\mathbf{v}=(\mathbf{v}[1], \ldots, \mathbf{v}[|\mathbf{v}|])$. A (binary) string $x$ is identified with a vector over $\{0,1\}$ so that $|x|$ is its length and $x[i]$ is its $i$-th bit. If $F: \mathcal{K} \times \mathcal{D} \rightarrow \mathcal{R}$ is a family of functions and $\mathbf{x}$ is a vector over $\mathcal{D}$ then $F(K, \mathbf{x})$ denotes the vector $(F(K, \mathbf{x}[1]), \ldots, F(K, \mathbf{x}[|\mathbf{x}|]))$. Read the term "efficient" as meaning "polynomial-time" in the natural asymptotic extension of our concrete framework.

GAMES. Some of our definitions and proofs are expressed via code-based games [6]. Recall that such a game - see Fig. 1 for an example- consists of an (optional) INITIALIZE procedure and procedures to respond to adversary oracle queries. A game $G$ is executed with an adversary $A$ as follows. First, Initialize (if present) executes. Then $A$ executes, its oracle queries being answered by the corresponding procedures of $G$. When $A$ terminates, its output, denoted $G^{A}$, is called the output of the game, and we let " $\mathrm{G}^{A} \Rightarrow 1$ " denote the event that this game output takes value 1 . Boolean flags are assumed initialized to false. The running time of an adversary by convention is the worst case time for the execution of the adversary with any of the games defining its security, so that the time of the called game procedures is included. When (as often) we describe a game in text and say the game "begins" by doing something, we are describing how INITIALIZE works.

PRFs. The advantage of an adversary $A$ in attacking the (standard) prf security of a family of functions $F: \mathcal{K} \times \mathcal{D} \rightarrow \mathcal{R}$ is defined via

$$
\mathbf{A d v}_{F}^{\mathrm{prf}}(A)=\operatorname{Pr}\left[\operatorname{PRFReal}_{F}^{A} \Rightarrow 1\right]-\operatorname{Pr}\left[\operatorname{PRFRand}_{F}^{A} \Rightarrow 1\right] .
$$

Game PRFReal $F$ begins by picking $K \stackrel{\$}{\leftarrow} \mathcal{K}$ and responds to oracle query $\mathrm{FN}(x)$ via $F(K, x)$. Game PRFRand $\operatorname{Pr}_{F}$ begins by picking $f \stackrel{\$}{\leftarrow} \operatorname{Fun}(\mathcal{D}, \mathcal{R})$ and responds to oracle query $\mathrm{FN}(x)$ via $f(x)$.

RKA-PRFs. We recall definitions from [5]. Let $F: \mathcal{K} \times \mathcal{D} \rightarrow \mathcal{R}$ be a family of functions and $\Phi \subseteq \operatorname{Fun}(\mathcal{K}, \mathcal{K})$. The members of $\Phi$ are called RKD (related-key deriving) functions. An adversary is said to be $\Phi$-restricted if its oracle queries $(\phi, x)$ satisfy $\phi \in \Phi$. The advantage of a $\Phi$-restricted adversary $A$ in attacking the prf-rka security of $F$ is defined via

$$
\mathbf{A d v}_{\Phi, F}^{\text {prf-rka }}(A)=\operatorname{Pr}\left[\operatorname{RKPRFReal}_{F}^{A} \Rightarrow 1\right]-\operatorname{Pr}\left[\operatorname{RKPRFRand}_{F}^{A} \Rightarrow 1\right] .
$$

Game RKPRFReal R $_{F}$ begins by picking $K \stackrel{\$}{\leftarrow} \mathcal{K}$ and responds to oracle query $\operatorname{RKFN}(\phi, x)$ via $F(\phi(K), x)$. Game RKPRFRand ${ }_{F}$ begins by picking $K \stackrel{\$}{\leftarrow} \mathcal{K}$ and $G \stackrel{\$}{\leftarrow} \operatorname{FF}(\mathcal{K}, \mathcal{D}, \mathcal{R})$, and responds to oracle query $\operatorname{RKFN}(\phi, x)$ via $G(\phi(K), x)$. $\mathrm{CR}$ HASH FUNCTIONS. The advantage of $C$ in attacking the $\mathrm{cr}$ (collision-resistance) security of $H: \mathcal{D} \rightarrow \mathcal{R}$ is

$$
\mathbf{A d v}_{H}^{\mathrm{cr}}(C)=\operatorname{Pr}\left[x \neq x^{\prime} \text { and } H(x)=H\left(x^{\prime}\right)\right]
$$

where the probability is over $\left(x, x^{\prime}\right) \stackrel{\$}{\leftarrow} C$. For simplicity and to better reflect practice, we view hash functions as unkeyed. This means there always exists an 
efficient $C$ whose cr-advantage is 1 , but that does not mean we can find it, and our results remain meaningful because the proofs give explicit constructions of cr-adversaries from other adversaries [52]. We could extend our treatment to let hash functions be families, which would be more rigorous. We can't make the hash key part of the PRF key because then it would be subject to the RKA, but since its secrecy is not needed for security, we can make it a public parameter. Thus, keyed hash functions require an extended syntax for function families in which functions in the family depended on a public parameter, and we have chosen to avoid this.

\section{Constructions of RKA-PRFs and RKA-PRPs}

In this section we describe and analyze our RKA-PRF constructions. We begin by defining the key-malleability and key fingerprint notions on which the general construction is based. Theorem 1 states the general construction and proves its security. Then we show how to instantiate the general construction to obtain DDH based RKA-PRFs for group-induced classes as well as other RKA-PRFs.

Key-Malleability. Suppose $M: \mathcal{K} \times \mathcal{D} \rightarrow \mathcal{R}$ is a family of functions and $\Phi \subseteq \operatorname{Fun}(\mathcal{K}, \mathcal{K})$ is a set of RKD functions. Suppose T is a deterministic algorithm that given an oracle $f: \mathcal{D} \rightarrow \mathcal{R}$ and inputs $(\phi, x) \in \Phi \times \mathcal{D}$ returns a point $\mathrm{T}^{f}(\phi, x) \in \mathcal{R}$. We say that $\mathrm{T}$ is a key-transformer for $(M, \Phi)$ if it satisfies two conditions. The first, called correctness, asks that $M(\phi(K), x)=\mathrm{T}^{M(K, \cdot)}(\phi, x)$ for every $(\phi, K, x) \in \Phi \times \mathcal{K} \times \mathcal{D}$. This is a relatively straightforward condition saying that one can compute $M(\phi(K), x)$ from $\phi, x$ if one has an oracle for $M(K, \cdot)$. The second condition, called uniformity, is more subtle. Roughly, it says that if the oracle provided to $\mathrm{T}$ is random then the outputs of $\mathrm{T}$ on any input sequence $\left(\phi_{1}, x_{1}\right), \ldots,\left(\phi_{q}, x_{q}\right)$ are uniformly and independently distributed as

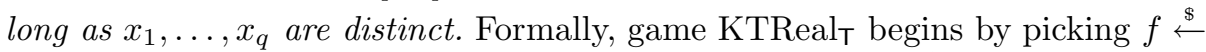
$\operatorname{Fun}(\mathcal{D}, \mathcal{R})$ and responds to oracle query $\operatorname{KTFN}(\phi, x)$ via $\operatorname{T}^{f}(\phi, x)$ while game $\operatorname{KTRand}_{\mathrm{T}}$ makes no initializations and responds to oracle query $\operatorname{KTFN}(\phi, x)$ by picking and returning a random point in $\mathcal{R}$. Let us say a $\Phi$-restricted adversary is unique input if, in its oracle queries $\left(\phi_{1}, x_{1}\right), \ldots,\left(\phi_{q}, x_{q}\right)$, the points $x_{1}, \ldots, x_{q}$ are always distinct, where by "always" we mean with probability one regardless of how oracle queries are answered and what are the coins of the adversary. The uniformity requirement is that

$$
\operatorname{Pr}\left[\operatorname{KTReal}_{\mathrm{T}}^{U} \Rightarrow 1\right]=\operatorname{Pr}\left[\operatorname{KTRand}_{\mathrm{T}}^{U} \Rightarrow 1\right]
$$

for every unique-input $\Phi$-restricted adversary $U$ against the uniformity of $\mathrm{T}$. We say $M$ is $\Phi$-key-malleable if there exists an efficient key transformer for $(M, \Phi)$.

That key-malleability might be useful to obtain RKA-PRFs is, on the one hand, intuitive, because the correctness property clearly allows us to simulate queries to $M(\phi(K), \cdot)$ via queries to $M(K, \cdot)$. It is, on the other hand, counterintuitive, because the same correctness property immediately yields an attack showing that $M$ is not a $\Phi$-RKA-PRF as long as $\Phi$ contains the identity function 
id and a function $\phi$ satisfying $\phi(K) \neq K$ for all $K \in \mathcal{K}$, conditions met by any group-induced $\Phi$. Indeed, consider $\Phi$-restricted adversary $A$ that, for some $x \in \mathcal{D}$, makes query $y \leftarrow \operatorname{RKFN}(\phi, x)$. Then it runs $\mathrm{T}$ on inputs $\phi, x$ to get an output $z$, answering any oracle query $w$ made in this computation by $\operatorname{RKFN}(\mathrm{id}, w)$. It returns 1 if $y=z$ and 0 otherwise. Correctness says that $A$ always returns 1 in game RKPRFReal $_{M}$. But the assumption on $\phi$ implies that $A$ returns 1 with probability at most $1 /|\mathcal{R}|$ in game $\operatorname{RKPRFRand}{ }_{M}$. $\operatorname{So} \operatorname{Adv}_{M, \Phi}^{\text {prf-rka }}(A)$ is almost 1 .

Although a key-malleable $M$ is not a $\Phi$-RKA-PRF, one can show that it is an RKA-PRF versus unique-input adversaries. (The adversary of the above attack need not be unique-input.) This leaves two questions. The first is how to bridge the gap to arbitrary adversaries, which we do via the concept of key fingerprints discussed below. The second is how to obtain key-malleable PRFs, which we will do later via the Naor-Reingold [47 and Lewko-Waters 43] constructs.

KEY FIngerprints. Suppose $M: \mathcal{K} \times \mathcal{D} \rightarrow \mathcal{R}$ is a family of functions and $\Phi \subseteq \operatorname{Fun}(\mathcal{K}, \mathcal{K})$ is a set of RKD functions. Let $\mathbf{w}$ be vector over $\mathcal{D}$ and let $m=|\mathbf{w}|$. We say that $\mathbf{w}$ is a key fingerprint for $(M, \Phi)$ if

$$
\begin{aligned}
& (M(\phi(K), \mathbf{w}[1]), \ldots, M(\phi(K), \mathbf{w}[m])) \\
& \neq\left(M\left(\phi^{\prime}(K), \mathbf{w}[1]\right), \ldots, M\left(\phi^{\prime}(K), \mathbf{w}[m]\right)\right)
\end{aligned}
$$

for all $K \in \mathcal{K}$ and all distinct $\phi, \phi^{\prime} \in \Phi$.

Let's call a class $\Phi \subseteq$ Fun $(\mathcal{K}, \mathcal{K})$ of RKD functions claw-free if $\phi(K) \neq \phi^{\prime}(K)$ for every key $K \in \mathcal{K}$ and every distinct $\phi, \phi^{\prime} \in \Phi[46] 5$. We note that if $(M, \Phi)$ has a key fingerprint then it follows automatically that $\Phi$ is claw-free. Indeed, if there is a $K$ and $\phi, \phi^{\prime}$ such that $\phi(K)=\phi^{\prime}(K)$ then there can be no $\mathbf{w}$ for which Equation (5) is true. We will use this frequently below.

We say that $\mathbf{w}$ is a strong key fingerprint for $(M, \Phi)$ if

$$
(M(K, \mathbf{w}[1]), \ldots, M(K, \mathbf{w}[m])) \neq\left(M\left(K^{\prime}, \mathbf{w}[1]\right), \ldots, M\left(K^{\prime}, \mathbf{w}[m]\right)\right)
$$

for all distinct $K, K^{\prime} \in \mathcal{K}$. If $\Phi$ is claw-free then a strong key fingerprint for $(M, \Phi)$ is also a key fingerprint for $(M, \Phi)$, which we will use in analyzing our constructs. If $\Phi$ is complete - recall this means that for every $K, K^{\prime} \in \mathcal{K}$ there is a $\phi \in \Phi$ such that $\phi(K)=K^{\prime}$ - then any key fingerprint for $(M, \Phi)$ is also a strong key fingerprint for $(M, \Phi)$. In general, however, the existence of a key fingerprint may not imply the existence of a strong key fingerprint.

Construction. Let $M: \mathcal{K} \times \mathcal{D} \rightarrow \mathcal{R}$ be a key-malleable family of functions and $\mathrm{T}$ a key transformer for $(M, \Phi)$. Let $\mathbf{w} \in \mathcal{D}^{m}$ be a key-fingerprint for $(M, \Phi)$. We say that a point $w \in \mathcal{D}$ is a possible oracle query for $\mathrm{T}$ relative to $(M, \Phi, \mathbf{w})$ if there exists $(f, \phi, i) \in \operatorname{Fun}(\mathcal{D}, \mathcal{R}) \times \Phi \times\{1, \ldots, m\}$ such that the computation $\mathrm{T}^{f}(\phi, \mathbf{w}[i])$ makes oracle query $w$. We let $\operatorname{Qrs}(\mathrm{T}, M, \Phi, \mathbf{w})$ be the set of all possible oracle queries $w$ for $\mathrm{T}$ relative to $(M, \Phi, \mathbf{w})$. Let $\overline{\mathcal{D}}=\mathcal{D} \times \mathcal{R}^{m}$. A hash function $H$ with domain $\overline{\mathcal{D}}$ is said to be compatible with $(\mathrm{T}, M, \Phi, \mathbf{w})$ if its range is $\mathcal{D} \backslash \operatorname{Qrs}(\mathbf{T}, M, \Phi, \mathbf{w})$. That is, possible oracle queries of $\mathrm{T}$ relative to $(M, \Phi, \mathbf{w})$ are not allowed to be outputs of $H$. With this, we can say what are the ingredients of our construction of a $\Phi$-RKA-PRF: (1) a $\Phi$-key-malleable PRF, meaning a 
family of functions $M: \mathcal{K} \times \mathcal{D} \rightarrow \mathcal{R}$ such that, on the one hand, $M$ is a PRF and, on the other hand, there exists a key transformer $\mathrm{T}$ for $(M, \Phi)$; $(2)$ a key fingerprint $\mathbf{w}$ for $(M, \Phi)$; and (3) a collision-resistant hash function $H: \overline{\mathcal{D}} \rightarrow$ $\mathcal{D} \backslash \mathrm{Qrs}(\mathrm{T}, M, \Phi, \mathbf{w})$ that is compatible with $(\mathrm{T}, M, \Phi, \mathbf{w})$. We combine them to build $F: \mathcal{K} \times \mathcal{D} \rightarrow \mathcal{R}$ that on input $K, x$ computes $\overline{\mathbf{w}} \leftarrow M(K, \mathbf{w})$-recall that, as per our notational conventions, $M(K, \mathbf{w})$ is the vector whose $i$-th component is $M(K, \mathbf{w}[i])$ for $1 \leq i \leq m-$ and then returns $M(K, H(x, \overline{\mathbf{w}}))$. The following theorem says that $F$ is a $\Phi$-RKA-PRF assuming $M$ is a PRF and $H$ is collisionresistant. No assumptions are made on $\Phi$ beyond those implied by the conditions stated here.

Theorem 1. Let $M: \mathcal{K} \times \mathcal{D} \rightarrow \mathcal{R}$ be a family of functions and $\Phi \subseteq \operatorname{Fun}(\mathcal{K}, \mathcal{K})$ a class of RKD functions. Let $\mathrm{T}$ be a key-transformer for $(M, \Phi)$ making $Q_{\mathrm{T}}$ oracle queries, and let $\mathbf{w} \in \mathcal{D}^{m}$ be a key fingerprint for $(M, \Phi)$. Let $\overline{\mathcal{D}}=\mathcal{D} \times \mathcal{R}^{m}$ and let $H: \overline{\mathcal{D}} \rightarrow S$ be a hash function that is compatible with $(\mathrm{T}, M, \Phi, \mathbf{w})$, so that $S=\mathcal{D} \backslash \operatorname{Qrs}(\mathrm{T}, M, \Phi, \mathbf{w})$. Define $F: \mathcal{K} \times \mathcal{D} \rightarrow \mathcal{R}$ by

$$
F(K, x)=M(K, H(x, M(K, \mathbf{w})))
$$

for all $K \in \mathcal{K}$ and $x \in \mathcal{D}$. Let $A$ be a $\Phi$-restricted adversary against the prfrka security of $F$ that makes $Q_{A} \leq|S|$ oracle queries. Then we can construct an adversary $B$ against the prf-security of $M$ and an adversary $C$ against the cr-security of $H$ such that

$$
\mathbf{A} \mathbf{d} \mathbf{v}_{\Phi, F}^{\text {prf-rka }}(A) \leq \mathbf{A} \mathbf{d} \mathbf{v}_{M}^{\mathrm{prf}}(B)+\mathbf{A} \mathbf{d} \mathbf{v}_{H}^{\mathrm{cr}}(C) .
$$

Adversary $B$ makes $(m+1) \cdot Q_{\top} Q_{A}$ oracle queries, and $B$ and $C$ have the same running time as $A$.

Proof (Theorem 11). We use the game sequence of Fig. 1 in the analysis below abbreviating by $W_{i}$ the event " $G_{i}^{A} \Rightarrow 1$ ". We assume (wlog) that $A$ never repeats an oracle query. Game $G_{0}$ simply instantiates game RKPRFReal $F$ of the definition of Section 2 with our construction $F$, so

$$
\operatorname{Pr}\left[\operatorname{RKPRFReal}_{F}^{A} \Rightarrow 1\right]=\operatorname{Pr}\left[W_{0}\right] .
$$

Game $G_{1}$, which does not include the boxed code, introduces some book-keeping, keeping track of hash values in a set $D$ and setting a flag bad to true if it ever sees a repeat. The book-keeping does not affect the values returned by RKFN so

$$
\operatorname{Pr}\left[W_{1}\right]=\operatorname{Pr}\left[W_{0}\right] .
$$

Game $G_{2}$ adds the boxed code which "corrects" a hash value repetition by picking instead a value that, being drawn from $S \backslash D$, will not repeat any previous one. The addition of this "artificial" step, leading to a game different from the "real" one, is to ensure that the values of $h$ on which $\mathrm{T}^{f}(\phi, h)$ is later called (lines $37,47,57)$ are distinct, putting us in a position to exploit the uniformity of $\mathrm{T}$ and replace the outputs by random values. This, however, is some distance away. For the moment we observe that games $G_{1}, G_{2}$ are identical until bad — differ only 


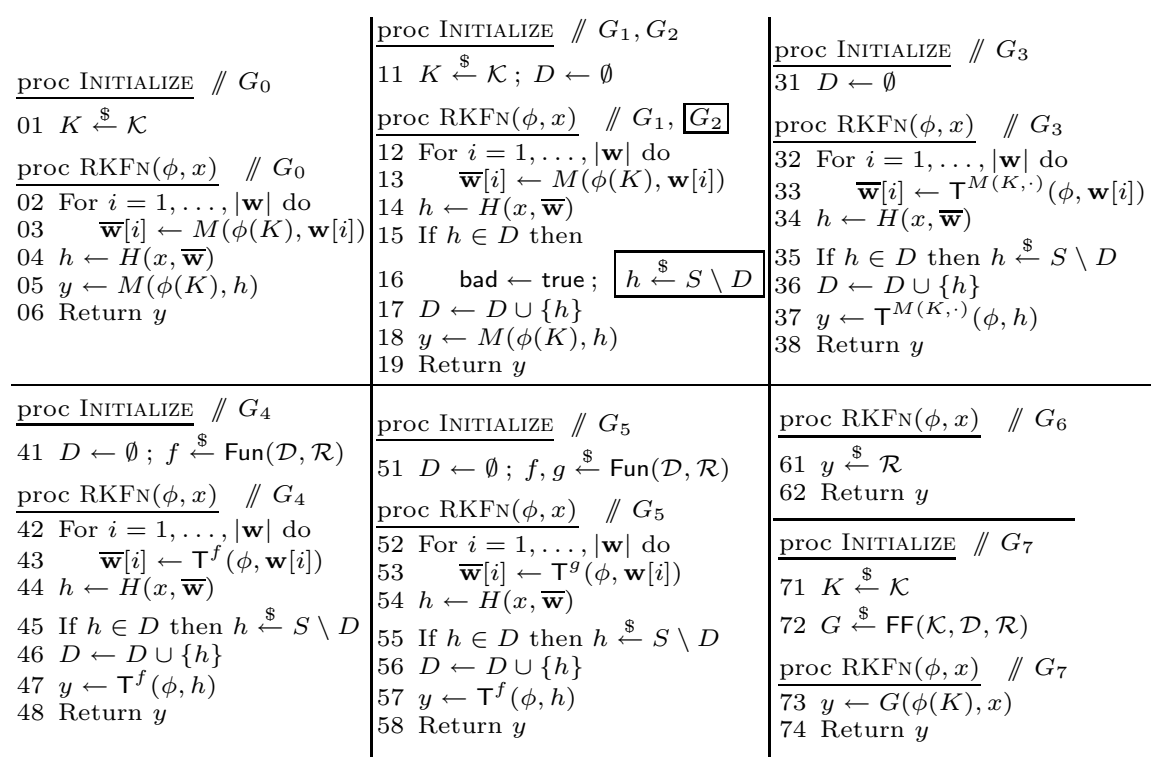

Fig. 1. Games for the proof of Theorem 1 Game $G_{2}$ includes the boxed code and game $G_{1}$ does not.

in code following the setting of bad to true - and hence the fundamental lemma of game playing [6] implies that

$$
\operatorname{Pr}\left[W_{1}\right] \leq \operatorname{Pr}\left[W_{2}\right]+\operatorname{Pr}\left[B_{1}\right]
$$

where $B_{1}$ denotes the event that the execution of $A$ with game $G_{1}$ sets the flag bad to true. Making crucial use of the assumption that $\mathbf{w}$ is a key fingerprint for $(M, \Phi)$, we design adversary $C$ attacking the cr-security of $H$ such that

$$
\operatorname{Pr}\left[B_{1}\right] \leq \mathbf{A d v}_{H}^{\mathrm{cr}}(C) .
$$

Adversary $C$ begins by picking $K \stackrel{\$}{\leftarrow} \mathcal{K}$ and initializing a counter $j \leftarrow 0$. It then runs $A$. When the latter makes a $\operatorname{RKFN-query~}(\phi, x)$, adversary $C$ responds via

$$
\begin{aligned}
& \text { For } i=1, \ldots,|\mathbf{w}| \text { do } \overline{\mathbf{w}}[i] \leftarrow M(\phi(K), \mathbf{w}[i]) \\
& j \leftarrow j+1 ; \phi_{j} \leftarrow \phi ; x_{j} \leftarrow x ; \overline{\mathbf{w}}_{j} \leftarrow \overline{\mathbf{w}} ; h_{j} \leftarrow H(x, \overline{\mathbf{w}}) ; y \leftarrow M(\phi(K), h) \\
& \text { Return } y
\end{aligned}
$$

When $A$ halts, $C$ searches for $a, b$ satisfying $1 \leq a<b \leq j$ such that $h_{a}=h_{b}$ and, if it finds them, outputs $\left(x_{a}, \overline{\mathbf{w}}_{a}\right),\left(x_{b}, \overline{\mathbf{w}}_{b}\right)$ and halts. Towards justifying Equation (12) the main question is, why are $\left(x_{a}, \overline{\mathbf{w}}_{a}\right),\left(x_{b}, \overline{\mathbf{w}}_{b}\right)$ distinct? The assumption that $A$ never repeats an oracle query means that $\left(\phi_{a}, x_{a}\right) \neq\left(\phi_{b}, x_{b}\right)$. Now consider two cases. First, if $\phi_{a}=\phi_{b}$ then we must have $x_{a} \neq x_{b}$ whence of course $\left(x_{a}, \overline{\mathbf{w}}_{a}\right) \neq\left(x_{b}, \overline{\mathbf{w}}_{b}\right)$. Second, if $\phi_{a} \neq \phi_{b}$ then the assumption that $\mathbf{w}$ is a key fingerprint for $(M, \Phi)$ means, by Equation (5), that $\overline{\mathbf{w}}_{a} \neq \overline{\mathbf{w}}_{b}$ and again $\left(x_{a}, \overline{\mathbf{w}}_{a}\right) \neq\left(x_{b}, \overline{\mathbf{w}}_{b}\right)$. 
In game $G_{3}$, we use the key transformer T, given by the assumed $\Phi$-keymalleability of $M$, to compute $M(\phi(K), \cdot)$ via oracle calls to $M(K, \cdot)$, both at line 33 and at line 37 . The correctness property of the key transformer implies

$$
\operatorname{Pr}\left[W_{2}\right]=\operatorname{Pr}\left[W_{3}\right] .
$$

Game $G_{4}$ replaces the oracle given to $\mathrm{T}$ by a random function. We design adversary $B$ attacking the prf-security of $M$ such that

$$
\operatorname{Pr}\left[W_{3}\right]-\operatorname{Pr}\left[W_{4}\right] \leq \mathbf{A d v}_{M}^{\mathrm{prf}}(B) .
$$

This is possible because the games make only oracle access to $M(K, \cdot)$ and $f$, respectively. In detail, adversary $B$ runs $A$. When the latter makes a RKFNquery $(\phi, x)$, adversary $B$ responds via

For $i=1, \ldots,|\mathbf{w}|$ do $\overline{\mathbf{w}}[i] \leftarrow \mathrm{T}^{\mathrm{FN}}(\phi, \mathbf{w}[i]) ; h \leftarrow H(x, \overline{\mathbf{w}}) ; y \leftarrow \mathrm{T}^{\mathrm{FN}}(\phi, h)$ Return $y$

where FN is $B$ 's own oracle. When $A$ halts, $B$ halts with the same output. Then

$$
\operatorname{Pr}\left[\operatorname{PRFReal}_{M}^{B} \Rightarrow 1\right]=\operatorname{Pr}\left[W_{3}\right] \quad \text { and } \quad \operatorname{Pr}\left[\operatorname{PRFRand}_{M}^{B} \Rightarrow 1\right]=\operatorname{Pr}\left[W_{4}\right]
$$

so Equation (14) follows from Equation (2).

Rather than return $y=\mathrm{T}^{f}(\phi, h)$ as at lines 47,48 , we would like to pick and return a random $y$, as at lines 61,62 of game $G_{6}$, saying this makes no difference by the uniformity of $\mathrm{T}$. But we have to be careful, because line 47 is not the only place $f$ is used in $G_{4}$. Oracle $f$ is also being queried in the computation $\mathrm{T}^{f}(\phi, \mathbf{w}[i])$ at line 43 , and if a $f$-query made here equals an input $h$ at line 47 , then it is unclear we can argue randomness of the line 47 output $y$ based on the uniformity of T. The assumed compatiblity of $H$ with $(\mathrm{T}, M, \Phi, \mathbf{w})$ comes to the rescue. It says the queries to $f$ in the computation $\mathrm{T}^{f}(\phi, \mathbf{w}[i])$ at line 43 , which fall within the set $\operatorname{Qrs}(\mathrm{T}, M, \Phi, \mathbf{w})$, are not in the set $S$ that is the range of $H$. Thus, the calls to $f$ at lines 43 and 47 can be answered with different, independent random functions without affecting the distribution of the procedure output. In other words, considering game $G_{5}$, which switches $f$ to $g$ at line 53 but not at line 57 , the compatibility of $H$ with $(\mathrm{T}, M, \Phi, \mathbf{w})$ implies that

$$
\operatorname{Pr}\left[W_{4}\right]=\operatorname{Pr}\left[W_{5}\right] .
$$

We will now exploit the uniformity of $\mathrm{T}$ to show that

$$
\operatorname{Pr}\left[W_{5}\right]=\operatorname{Pr}\left[W_{6}\right] .
$$

To do this we design unique-input $\Phi$-restricted adversary $U$ against the uniformity of $\mathrm{T}$ such that

$$
\operatorname{Pr}\left[\operatorname{KTReal}_{M}^{U} \Rightarrow 1\right]=\operatorname{Pr}\left[W_{5}\right] \text { and } \operatorname{Pr}\left[\mathrm{KTRand}_{M}^{U} \Rightarrow 1\right]=\operatorname{Pr}\left[W_{6}\right] \text {. }
$$

Equation (16) follows from Equation (4). Adversary $U$ begins by initializing set $D \leftarrow \emptyset$ and picking $g \stackrel{\$}{\leftarrow} \operatorname{Fun}(\mathcal{D}, \mathcal{R})$. (Adversary $U$ of the uniformity condition is not required to be efficient so picking $g$ like this is okay but in any case we could make $U$ efficient if we liked by simulating $g$ via lazy sampling rather than 


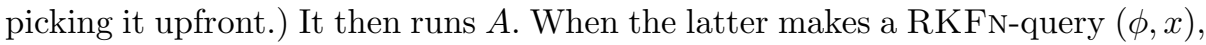
adversary $U$ responds via

$$
\begin{aligned}
& \text { For } i=1, \ldots,|\mathbf{w}| \text { do } \overline{\mathbf{w}}[i] \leftarrow \mathrm{T}^{g}(\phi, \mathbf{w}[i]) \\
& j \leftarrow j+1 ; \phi_{j} \leftarrow \phi ; h_{j} \leftarrow H(x, \overline{\mathbf{w}}) ; \text { If } h_{j} \in D \text { then } h_{j} \stackrel{\$}{\leftarrow} \backslash D \\
& y \leftarrow \operatorname{KTFN}\left(\phi_{j}, h_{j}\right) ; \operatorname{Return} y
\end{aligned}
$$

where KTFN is $U$ 's own oracle. The delicate question is, why is $U$ unique-input? The boxed code introduced at line 16, carried through to line 55, and reflected by the "If" statement in the code for $U$ above, ensures that $h_{1}, \ldots, h_{j}$ are all distinct at the end of $U$ 's computation as long as $Q_{A} \leq|S|$, which the theorem assumed. Equation (17) follows.

The claw-freeness of $\Phi$ - recall this follows from the assumption that $(M, \Phi)$ has a key fingerprint - implies that if $(\phi, x) \neq\left(\phi^{\prime}, x^{\prime}\right)$ then $(\phi(K), x) \neq\left(\phi^{\prime}(K), x^{\prime}\right)$. This together with the assumption that $A$ does not repeat an oracle query imply

$$
\operatorname{Pr}\left[W_{6}\right]=\operatorname{Pr}\left[W_{7}\right]=\operatorname{Pr}\left[\operatorname{RKPRFRand}_{F}^{A} \Rightarrow 1\right] .
$$

Equation (8) follows from Equations (9), (10), (11), (12), (13), (14), (15), (16), (18), (3).

OUR MUltiplicAtive DDH-BASED RKA-PRF. We instantiate our general construction to get a DDH-based $\Phi$-RKA-PRF where $\Phi$ is group induced. Let $\mathbb{G}$ be a (multiplicatively written) group of prime order $p$, and let $g \in \mathbb{G}$ be an arbitrary generator of $\mathbb{G}$. The classic Naor-Reingold 47] PRF NR: $\mathbb{Z}_{p}^{n+1} \times\{0,1\}^{n} \rightarrow$ $\mathbb{G}$ is defined via

$$
\mathrm{NR}(\mathbf{a}, x)=g^{\mathbf{a}[0] \prod_{i=1}^{n} \mathbf{a}[i]^{x[i]}}
$$

for all $\mathbf{a} \in \mathbb{Z}_{p}^{n+1}$ and $x \in\{0,1\}^{n}$. Recall the advantage of an adversary $B$ against the DDH problem in $\mathbb{G}$ is

$$
\mathbf{A d v}_{\mathbb{G}}^{\mathrm{ddh}}(B)=\operatorname{Pr}\left[B\left(g^{a}, g^{b}, g^{a b}\right) \Rightarrow 1\right]-\operatorname{Pr}\left[B\left(g^{a}, g^{b}, g^{c}\right) \Rightarrow 1\right],
$$

where the probabilities are over $a, b, c \stackrel{\$}{\leftarrow} \mathbb{Z}_{p}^{*}$. The following result of [47] says that $\mathrm{NR}$ is a PRF if DDH is hard in $\mathbb{G}$.

Lemma 2. 47] Let $\mathbb{G}=\langle g\rangle$ be a group of prime order $p$ and $\mathrm{NR}: \mathbb{Z}_{p}^{n+1} \times$ $\{0,1\}^{n} \rightarrow \mathbb{G}$ the family of functions defined via Equation (19). Let $A$ an adversary against the prf-security of $\mathrm{NR}$ that makes $Q$ oracle queries. Then we can construct an adversary $B$ against the DDH problem in $\mathbb{G}$ such that

$$
\mathbf{A d v}_{\mathrm{NR}}^{\mathrm{prf}}(A) \leq n \cdot \mathbf{A} \mathbf{d} \mathbf{v}_{\mathbb{G}}^{\mathrm{ddh}}(B) .
$$

The running time of $B$ is that of $A$ plus the time required for $\mathcal{O}(Q)$ exponentiations in $\mathbb{G}$.

Group-induced class: Define operation $*$ by $\mathbf{a} * \mathbf{d}=(\mathbf{a}[0] \mathbf{d}[0], \ldots, \mathbf{a}[n] \mathbf{d}[n])$ where operations on components are multiplications modulo $p$. Then the set $\mathcal{K}=$ $\left(\mathbb{Z}_{p}^{*}\right)^{n+1}$ is a group under $*$. Let $\phi_{\mathbf{d}}^{*}: \mathcal{K} \rightarrow \mathcal{K}$ be defined by $\phi_{\mathbf{d}}^{*}(\mathbf{a})=\mathbf{a} * \mathbf{d}$ 
for all $\mathbf{a}, \mathbf{d} \in \mathcal{K}$. Let $\Phi=\operatorname{rkd}\left[\left(\mathbb{Z}_{p}^{*}\right)^{n+1}, *\right]$ be the class of all $\phi_{\mathbf{d}}^{*}$ as $\mathbf{d}$ ranges over $\mathcal{K}$. This class is group-induced, the group being $(\mathcal{K}, *)$.

Key malleability: We claim that NR is $\Phi$-key-malleable. The key-transformer

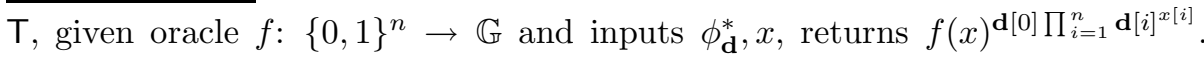
Correctness holds because

$$
\operatorname{T}^{\mathrm{NR}(\mathbf{a}, \cdot)}\left(\phi_{\mathbf{d}}^{*}, x\right)=\mathrm{NR}(\mathbf{a}, x)^{\mathbf{d}[0] \prod_{i=1}^{n} \mathbf{d}[i]^{x[i]}}=\mathrm{NR}(\mathbf{a} * \mathbf{d}, x) .
$$

In game $\mathrm{KTReal}_{\mathrm{NR}}$, the responses received by unique-input, $\Phi$-restricted adversary $U$ to KTFN-queries $\left(\phi_{\mathbf{d}_{1}}^{*}, x_{1}\right), \ldots,\left(\phi_{\mathbf{d}_{q}}^{*}, x_{q}\right)$ are $f\left(x_{1}\right)^{\mathbf{d}_{1}[0] \prod_{i=1}^{n} \mathbf{d}_{1}[i]^{x[i]}}, \ldots$, $f\left(x_{q}\right)^{\mathbf{d}_{q}[0] \prod_{i=1}^{n} \mathbf{d}_{q}[i]^{x[i]}}$ where $f \stackrel{\$}{\leftarrow} \operatorname{Fun}\left(\{0,1\}^{n}, \mathbb{G}\right)$ was chosen by the game. Since $x_{1}, \ldots, x_{q}$ are distinct and the exponents are non-zero, these responses are randomly and independently distributed over $\mathbb{G}$. We have verified the uniformity condition.

Key fingerprint: For $i=1, \ldots, n$ let $\mathbf{w}[i]=0^{i-1}\|1\| 0^{n-i}$ be the string that is $\overline{\text { all zeros except }}$ at position $i$, where it has a one. Let $\mathbf{w}[0]=0^{n}$. We claim that $\mathbf{w}$ is a strong key fingerprint for $(\mathrm{NR}, \Phi)$. To see this, first note that $(\mathrm{NR}(\mathbf{a}, \mathbf{w}[0]), \operatorname{NR}(\mathbf{a}, \mathbf{w}[1]) \ldots, \mathrm{NR}(\mathbf{a}, \mathbf{w}[n]))=\left(g^{\mathbf{a}[0]}, g^{\mathbf{a}[0] \mathbf{a}[1]}, \ldots, g^{\mathbf{a}[0] \mathbf{a}[n]}\right)$. Now if $\mathbf{a}, \mathbf{a}^{\prime} \in \mathcal{K}$ are distinct keys and $\mathbf{a}[0] \neq \mathbf{a}^{\prime}[0]$ then $g^{\mathbf{a}[0]} \neq g^{\mathbf{a}^{\prime}[0]}$. On the other hand if $\mathbf{a}[0]=\mathbf{a}^{\prime}[0]$ and $\mathbf{a}[i] \neq \mathbf{a}^{\prime}[i]$ for some $i>0$, then $g^{\mathbf{a}[0] \mathbf{a}[i]} \neq g^{\mathbf{a}^{\prime}[0] \mathbf{a}^{\prime}[i]}$. The claim follows from the definition of Equation (6) with $M=\mathrm{NR}$. Since $\Phi$ is claw-free, w is also a key fingerprint for $(\mathrm{NR}, \Phi)$, satisfying Equation (5) with $M=\mathrm{NR}$.

Compatible hash function: The set of possible oracle queries of $\mathrm{T}$ relative to

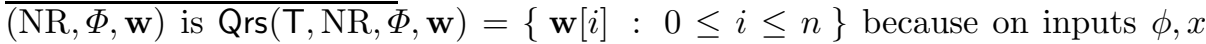
the only oracle query made by $\mathrm{T}$ is $x$ itself. Let $\overline{\mathcal{D}}=\{0,1\}^{n} \times \mathbb{G}^{n+1}$. If $h: \overline{\mathcal{D}} \rightarrow$ $\{0,1\}^{n-2}$ is collision resistant, then $H: \overline{\mathcal{D}} \rightarrow\{0,1\}^{n} \backslash \operatorname{Qrs}(\mathrm{T}, \mathrm{NR}, \Phi, \mathbf{w})$ defined by $H(x, \mathbf{z})=11 \| h(x, \mathbf{z})$ is collision resistant and compatible with $(\mathrm{T}, \mathrm{NR}, \Phi, \mathbf{w})$ because all members of $\operatorname{Qrs}(\mathrm{T}, \mathrm{NR}, \Phi, \mathbf{w})$ have Hamming weight at most 1 while outputs of $H$ have Hamming weight at least 2 .

We have all the ingredients. The following theorem combines the above with Theorem 1 and Lemma 2 to present our DDH-based $\Phi$-RKA-PRF for groupinduced $\Phi$ and specify its security.

Theorem 3. Let $\mathbb{G}=\langle g\rangle$ be a group of prime order $p$ and $\mathrm{NR}: \mathbb{Z}_{p}^{n+1} \times\{0,1\}^{n} \rightarrow$ $\mathbb{G}$ the family of functions defined via Equation (19). Let $\overline{\mathcal{D}}=\{0,1\}^{n} \times \mathbb{G}^{n+1}$ and let $h: \overline{\mathcal{D}} \rightarrow\{0,1\}^{n-2}$ be a hash function. Define $F:\left(\mathbb{Z}_{p}^{*}\right)^{n+1} \times\{0,1\}^{n} \rightarrow \mathbb{G}$ by

$$
F(\mathbf{a}, x)=\operatorname{NR}\left(\mathbf{a}, 11 \| h\left(x,\left(g^{\mathbf{a}[0]}, g^{\mathbf{a}[0] \mathbf{a}[1]}, \ldots, g^{\mathbf{a}[0] \mathbf{a}[n]}\right)\right)\right)
$$

for all $\mathbf{a} \in\left(\mathbb{Z}_{p}^{*}\right)^{n+1}$ and $x \in\{0,1\}^{n}$. Let $\Phi=\operatorname{rkd}\left[\left(\mathbb{Z}_{p}^{*}\right)^{n+1}\right.$, * where $*$ is the operation of component-wise multiplication modulo p. Let $A$ be a $\Phi$-restricted adversary against the prf-rka security of $F$ that makes $Q_{A} \leq 2^{n-2}$ oracle queries. Then we can construct an adversary $B$ against the DDH problem in $\mathbb{G}$ and an adversary $C$ against the cr-security of $h$ such that

$$
\mathbf{A d}_{\Phi, F}^{\mathrm{prf}-\mathrm{rka}}(A) \leq n \cdot \mathbf{A} \mathbf{d} \mathbf{v}_{\mathbb{G}}^{\mathrm{ddh}}(B)+\mathbf{A} \mathbf{d} \mathbf{v}_{h}^{\mathrm{cr}}(C)
$$


The running time of $B$ is that of $A$ plus the time required for $\mathcal{O}(n Q)$ exponentiations in $\mathbb{G}$. $C$ has the same running time as $A$.

A DLIN-BASED RKA-PRF. There are groups where DDH is easy but the DLIN problem [22] still seems hard, which motivated Lewko and Waters [43] to find a DLIN-based PRF. In the same vein, we seek a DLIN-based RKA-PRF.

Let $\mathbb{G}=\langle g\rangle$ be a group of prime order $p$. Lewko and Waters 43 ] describe their DLIN-based PRF as having key a randomly chosen tuple $\left(y_{0}, z_{0}, y_{1}, z_{1}, w_{1}, v_{1}\right.$, $\left.\ldots, y_{n}, z_{n}, w_{n}, v_{n}\right) \in \mathbb{Z}_{p}^{4 n+2}$ and then on input $x \in\{0,1\}^{n}$ computing its output as follows. Set $a \leftarrow y_{0} ; b \leftarrow z_{0}$ and then for $i=1, \ldots, n$ execute "If $x[i]=1$ then $a \leftarrow a y_{i}+b z_{i} ; b \leftarrow a w_{i}+b v_{i}$." Finally, return $g^{a}$. Lewko and Waters [43, Section 1] comment that "the additional complexity required to accomodate the weaker assumptions means that our functions can no longer be described by closed-form formulas like ...," referring, in the "...," to the formula for NR that we have given as Equation (19). We provide such a closed-form formula based on matrices. (This will put us in a position, via a slight modification of the construction, to apply Theorem 1 and obtain a RKA-PRF.) Let $\mathrm{AL}_{2}(p)$ denote the set of all 2 by 2 matrices over $\mathbb{Z}_{p}$. If $\mathbf{M} \in \mathrm{AL}_{2}(p)$ and $b \in\{0,1\}$ then $\mathbf{M}^{b}$ is the identity matrix if $b=0$ and is of course just $\mathbf{M}$ if $b=1$. If $\mathbf{u}=(\mathbf{u}[1], \mathbf{u}[2])$ is a 2 -vector over $\mathbb{Z}_{p}$ then $\mathbf{u} \cdot \mathbf{M}$ denotes the 2-vector obtained by the vector-matrix product in which $\mathbf{u}$ is viewed as a 1 by 2 matrix. We define $\mathrm{LW}: \mathrm{AL}_{2}(p)^{n+1} \times\{0,1\}^{n} \rightarrow \mathbb{G}$ via

$$
\operatorname{LW}(\mathbf{A}, x)=g^{\mathbf{y}[1]} \quad \text { where } \quad \mathbf{y}=(1,0) \cdot \mathbf{A}[0] \prod_{i=1}^{n} \mathbf{A}[i]^{x[i]}
$$

for all $\mathbf{A} \in \mathrm{AL}_{2}(p)^{n+1}$ and $x \in\{0,1\}^{n}$. Here the key is an $(n+1)$-vector $\mathbf{A}=$ $(\mathbf{A}[0], \ldots, \mathbf{A}[n])$ of 2 by 2 matrices over $\mathbb{Z}_{p}$. The formula left-multiplies the matrix product by the 2 -vector $(1,0)$ to get a 2 -vector $\mathbf{y}$ whose first component $\mathbf{y}[1]$ becomes the exponent to which $g$ is raised to get the function output. We claim LW is exactly the function described by the code above. (To verify this it helps to recall that matrix multiplication is associative. Strictly speaking the LW key is longer, being $4 n+4$ elements of $\mathbb{Z}_{p}$, but the second row of $\mathbf{A}[0]$ is effectively unused due to the product with $(1,0)$ so the effective key is $4 n+2$ points in $\mathbb{Z}_{p}$, as in the original construct.) Comparing with Equation (19), the closed-form formulation of Equation (21) makes clearer how LW is an analogue of NR.

To obtain a key-malleable PRF admitting a key fingerprint, we need two modifications. (The modifications are in fact to get the key fingerprint, not the key malleability.) First, we restrict the keyspace, drawing the matrices from $\mathrm{GL}_{2}(p) \subset \mathrm{AL}_{2}(p)$ rather than $\mathrm{AL}_{2}(p)$, where $\mathrm{GL}_{2}(p)$ is the set of invertible matrices in $\mathrm{AL}_{2}(p)$, usually referred to as the general linear group. Second, if $\mathbf{y}[1]=0$, we use $\mathbf{y}[2]$, which we will be able to guarantee is not 0 in this case, in its place. In detail, define $\mathrm{LW}^{*}: \mathrm{GL}_{2}(p)^{n+1} \times\{0,1\}^{n} \rightarrow \mathbb{G}$ via

$$
\mathrm{LW}^{*}(\mathbf{A}, x)=\left\{\begin{array}{l}
g^{\mathbf{y}[1]} \text { if } \mathbf{y}[1] \neq 0 \\
g^{\mathbf{y}[2]} \text { otherwise }
\end{array} \text { where } \mathbf{y}=(1,0) \cdot \mathbf{A}[0] \prod_{i=1}^{n} \mathbf{A}[i]^{x[i]}\right.
$$

for all $\mathbf{A} \in \mathrm{GL}_{2}(p)^{n+1}$ and $x \in\{0,1\}^{n}$. In 4 . we show how to apply our framework of Theorem 1 with $\mathrm{LW}^{*}$ in the role of $M$, obtaining our DLIN-based RKA-PRF. 
An ADDITIVE DDH-BASED RKA-PRF. The group $\left(\mathbb{Z}_{p}^{*}\right)^{n+1}$ of our multiplicative DDH-based RKA-PRF is multiplicative. Our final construct is a rkd $\left[\mathbb{Z}_{p}^{n},+\right]$ RKA-PRF, still DDH-based, where + is componentwise addition, so that the group is additive. This takes more work than the multiplicative construct. We were not able to work with NR itself but had to slightly modify it. The key transformer and proof of uniformity are more complex. The materiel is in [4].

From RKA-PRFs To RKA-PRPs. Cryptanalytic interest has mostly been in RKA-secure blockciphers, meaning, families of permutations. It is not clear how one might directly modify the constructions of Section 3 , which are families of functions, to make them families of permutations. We use, instead, a simple but powerful composition approach that produces a $\Phi$-RKA-PRP from a given $\Phi$-RKA-PRG and an ordinary PRP. We obtain appropriate $\Phi$-RKAPRGs by combining our $\Phi$-RKA-PRFs with deterministic extractors [26 3024]. This approach not only yields RKA-secure PRPs under chosen-plaintext attack (CPA) but even under chosen-ciphertext attack (CCA). In [4] we provide formal definitions for PRPs, RKA-PRPs and RKA-PRGs and state and prove the composition results.

\section{Acknowledgments}

We thank Xavier Boyen and Damien Vergnaud for explaining that the q-BDHIbased proof of PRF-security of BBDY from [27/21] only works when the domain of the function is small, rendering abortive our attempts to obtain RKA-security via this function. We thank Mira Belenkiy for suggesting that BBDY might help for RKA-security in the first place. We thank Mira and Tolga Acar for discussions on the practical relevance of RKAs that rekindled the first author's interest in this area.

\section{References}

1. Applebaum, B.: Fast cryptographic primitives based on the hardness of decoding random linear code. Technical Report TR-845-08, Princeton University (2008)

2. Bellare, M.: New proofs for NMAC and HMAC: Security without collisionresistance. In: Dwork, C. (ed.) CRYPTO 2006. LNCS, vol. 4117, pp. 602-619. Springer, Heidelberg (2006)

3. Bellare, M., Canetti, R., Krawczyk, H.: Keying hash functions for message authentication. In: Koblitz, N. (ed.) CRYPTO 1996. LNCS, vol. 1109, pp. 1-15. Springer, Heidelberg (1996)

4. Bellare, M., Cash, D.: Pseudorandom functions and permutations provablysecure against related key attacks. Cryptology ePrint Archive (2010) (full version of this abstract)

5. Bellare, M., Kohno, T.: A theoretical treatment of related-key attacks: RKA-PRPs, RKA-PRFs, and applications. In: Biham, E. (ed.) EUROCRYPT 2003. LNCS, vol. 2656, pp. 491-506. Springer, Heidelberg (2003)

6. Bellare, M., Rogaway, P.: The security of triple encryption and a framework for code-based game-playing proofs. In: Vaudenay, S. (ed.) EUROCRYPT 2006. LNCS, vol. 4004, pp. 409-426. Springer, Heidelberg (2006) 
7. Biham, E.: New types of cryptoanalytic attacks using related keys (extended abstract). In: Helleseth, T. (ed.) EUROCRYPT 1993. LNCS, vol. 765, pp. 398-409. Springer, Heidelberg (1994)

8. Biham, E.: New types of cryptanalytic attacks using related keys. Journal of Cryptology 7(4), 229-246 (1994)

9. Biham, E., Dunkelman, O., Keller, N.: Related-key boomerang and rectangle attacks. In: Cramer, R. (ed.) EUROCRYPT 2005. LNCS, vol. 3494, pp. 507-525. Springer, Heidelberg (2005)

10. Biham, E., Dunkelman, O., Keller, N.: A related-key rectangle attack on the full KASUMI. In: Roy, B. (ed.) ASIACRYPT 2005. LNCS, vol. 3788, pp. 443-461. Springer, Heidelberg (2005)

11. Biham, E., Dunkelman, O., Keller, N.: Related-key impossible differential attacks on 8-round AES-192. In: Pointcheval, D. (ed.) CT-RSA 2006. LNCS, vol. 3860, pp. 21-33. Springer, Heidelberg (2006)

12. Biham, E., Dunkelman, O., Keller, N.: A simple related-key attack on the full SHACAL-1. In: Abe, M. (ed.) CT-RSA 2007. LNCS, vol. 4377, pp. 20-30. Springer, Heidelberg (2006)

13. Biham, E., Dunkelman, O., Keller, N.: A unified approach to related-key attacks. In: Nyberg, K. (ed.) FSE 2008. LNCS, vol. 5086, pp. 73-96. Springer, Heidelberg (2008)

14. Biham, E., Shamir, A.: Differential fault analysis of secret key cryptosystems. In: Kaliski Jr., B.S. (ed.) CRYPTO 1997. LNCS, vol. 1294, pp. 513-525. Springer, Heidelberg (1997)

15. Biryukov, A., Dunkelman, O., Keller, N., Khovratovich, D., Shamir, A.: Key recovery attacks of practical complexity on AES variants with up to 10 rounds. In: Gilbert, H. (ed.) EUROCRYPT 2010. LNCS, vol. 6110, pp. 299-319. Springer, Heidelberg (2010)

16. Biryukov, A., Khovratovich, D.: Related-key cryptanalysis of the full AES-192 and AES-256. In: Matsui, M. (ed.) ASIACRYPT 2009. LNCS, vol. 5912, pp. 1-18. Springer, Heidelberg (2009)

17. Biryukov, A., Khovratovich, D., Nikolic, I.: Distinguisher and related-key attack on the full AES-256. In: Halevi, S. (ed.) CRYPTO 2009. LNCS, vol. 5677, pp. 231-249. Springer, Heidelberg (2009)

18. Black, J.: The ideal-cipher model, revisited: An uninstantiable blockcipher-based hash function. In: Robshaw, M.J.B. (ed.) FSE 2006. LNCS, vol. 4047, pp. 328-340. Springer, Heidelberg (2006)

19. Blunden, M., Escott, A.: Related key attacks on reduced round KASUMI. In: Matsui, M. (ed.) FSE 2001. LNCS, vol. 2355, pp. 277-285. Springer, Heidelberg (2002)

20. Boneh, D., Boyen, X.: Efficient selective-ID secure identity based encryption without random oracles. In: Cachin, C., Camenisch, J.L. (eds.) EUROCRYPT 2004. LNCS, vol. 3027, pp. 223-238. Springer, Heidelberg (2004)

21. Boneh, D., Boyen, X.: Short signatures without random oracles. In: Cachin, C., Camenisch, J.L. (eds.) EUROCRYPT 2004. LNCS, vol. 3027, pp. 56-73. Springer, Heidelberg (2004)

22. Boneh, D., Boyen, X., Shacham, H.: Short group signatures. In: Franklin, M. (ed.) CRYPTO 2004. LNCS, vol. 3152, pp. 41-55. Springer, Heidelberg (2004)

23. Boneh, D., DeMillo, R.A., Lipton, R.J.: On the importance of checking cryptographic protocols for faults (extended abstract). In: Fumy, W. (ed.) EUROCRYPT 1997. LNCS, vol. 1233, pp. 37-51. Springer, Heidelberg (1997) 
24. Canetti, R., Friedlander, J.B., Konyagin, S.V., Larsen, M., Lieman, D., Shparlinski, I.: On the statistical properties of Diffie-Hellman distributions. Israel J. Math. 120, 23-46 (2000)

25. Canetti, R., Goldreich, O., Halevi, S.: The random oracle methodology, revisited (preliminary version). In: 30th ACM STOC, pp. 209-218. ACM Press, New York (May 1998)

26. Chevalier, C., Fouque, P.-A., Pointcheval, D., Zimmer, S.: Optimal randomness extraction from a Diffie-Hellman element. In: Joux, A. (ed.) EUROCRYPT 2009. LNCS, vol. 5479, pp. 572-589. Springer, Heidelberg (2010)

27. Dodis, Y., Yampolskiy, A.: A verifiable random function with short proofs and keys. In: Vaudenay, S. (ed.) PKC 2005. LNCS, vol. 3386, pp. 416-431. Springer, Heidelberg (2005)

28. Dolev, D., Dwork, C., Naor, M.: Nonmalleable cryptography. SIAM Journal on Computing 30(2), 391-437 (2000)

29. Dunkelman, O., Keller, N., Kim, J.: Related-key rectangle attack on the full SHACAL-1. In: Biham, E., Youssef, A.M. (eds.) SAC 2006. LNCS, vol. 4356, pp. 28-44. Springer, Heidelberg (2007)

30. Fouque, P.-A., Pointcheval, D., Stern, J., Zimmer, S.: Hardness of distinguishing the MSB or LSB of secret keys in Diffie-Hellman schemes. In: Bugliesi, M., Preneel, B., Sassone, V., Wegener, I. (eds.) ICALP 2006. LNCS, vol. 4052, pp. 240-251. Springer, Heidelberg (2006)

31. Gennaro, R., Lysyanskaya, A., Malkin, T., Micali, S., Rabin, T.: Algorithmic tamper-proof (ATP) security: Theoretical foundations for security against hardware tampering. In: Naor, M. (ed.) TCC 2004. LNCS, vol. 2951, pp. 258-277. Springer, Heidelberg (2004)

32. Goldenberg, D., Liskov, M.: On related-secret pseudorandomness. In: Micciancio, D. (ed.) TCC 2010. LNCS, vol. 5978, pp. 255-272. Springer, Heidelberg (2010)

33. Goldreich, O., Goldwasser, S., Micali, S.: How to construct random functions. Journal of the ACM 33, 792-807 (1986)

34. Hong, S., Kim, J., Lee, S., Preneel, B.: Related-key rectangle attacks on reduced versions of SHACAL-1 and AES-192. In: Gilbert, H., Handschuh, H. (eds.) FSE 2005. LNCS, vol. 3557, pp. 368-383. Springer, Heidelberg (2005)

35. Iwata, T., Kohno, T.: New security proofs for the 3GPP confidentiality and integrity algorithms. In: Roy, B., Meier, W. (eds.) FSE 2004. LNCS, vol. 3017, pp. 427-445. Springer, Heidelberg (2004)

36. Jakimoski, G., Desmedt, Y.: Related-key differential cryptanalysis of 192-bit key AES variants. In: Matsui, M., Zuccherato, R.J. (eds.) SAC 2003. LNCS, vol. 3006, pp. 208-221. Springer, Heidelberg (2004)

37. Jaulmes, É., Joux, A., Valette, F.: On the security of randomized CBC-MAC beyond the birthday paradox limit: A new construction. In: Daemen, J., Rijmen, V. (eds.) FSE 2002. LNCS, vol. 2365, pp. 237-251. Springer, Heidelberg (2002)

38. Kelsey, J., Schneier, B., Wagner, D.: Related-key cryptanalysis of 3-WAY, BihamDES, CAST, DES-X, NewDES, RC2, and TEA. In: Han, Y., Okamoto, T., Qing, S. (eds.) ICICS 1997. LNCS, vol. 1334, pp. 233-246. Springer, Heidelberg (1997)

39. Kim, J., Hong, S., Preneel, B.: Related-key rectangle attacks on reduced AES-192 and AES-256. In: Biryukov, A. (ed.) FSE 2007. LNCS, vol. 4593, pp. 225-241. Springer, Heidelberg (2007)

40. Knudsen, L.R.: Cryptanalysis of LOKI91. In: Zheng, Y., Seberry, J. (eds.) AUSCRYPT 1992. LNCS, vol. 718, pp. 196-208. Springer, Heidelberg (1993)

41. Knudsen, L.R., Kohno, T.: Analysis of RMAC. In: Johansson, T. (ed.) FSE 2003. LNCS, vol. 2887, pp. 182-191. Springer, Heidelberg (2003) 
42. Ko, Y., Hong, S., Lee, W., Lee, S., Kang, J.-S.: Related key differential attacks on 27 rounds of XTEA and full-round GOST. In: Roy, B., Meier, W. (eds.) FSE 2004. LNCS, vol. 3017, pp. 299-316. Springer, Heidelberg (2004)

43. Lewko, A.B., Waters, B.: Efficient pseudorandom functions from the decisional linear assumption and weaker variants. In: Al-Shaer, E., Jha, S., Keromytis, A.D. (eds.) ACM CCS 2009, pp. 112-120. ACM Press, New York (November 2009)

44. Liskov, M., Rivest, R.L., Wagner, D.: Tweakable block ciphers. In: Yung, M. (ed.) CRYPTO 2002. LNCS, vol. 2442, pp. 31-46. Springer, Heidelberg (2002)

45. Luby, M., Rackoff, C.: How to construct pseudorandom permutations from pseudorandom functions. SIAM Journal on Computing 17(2) (1988)

46. Lucks, S.: Ciphers secure against related-key attacks. In: Roy, B., Meier, W. (eds.) FSE 2004. LNCS, vol. 3017, pp. 359-370. Springer, Heidelberg (2004)

47. Naor, M., Reingold, O.: Number-theoretic constructions of efficient pseudo-random functions. Journal of the ACM 51(2), 231-262 (2004)

48. Peikert, C., Waters, B.: Lossy trapdoor functions and their applications. In: Ladner, R.E., Dwork, C. (eds.) 40th ACM STOC, pp. 187-196. ACM Press, New York (2008)

49. Phan, R.C.-W.: Related-key attacks on triple-DES and DESX variants. In: Okamoto, T. (ed.) CT-RSA 2004. LNCS, vol. 2964, pp. 15-24. Springer, Heidelberg (2004)

50. Preneel, B., Govaerts, R., Vandewalle, J.: Hash functions based on block ciphers: A synthetic approach. In: Stinson, D.R. (ed.) CRYPTO 1993. LNCS, vol. 773, pp. 368-378. Springer, Heidelberg (1994)

51. Rogaway, P.: Efficient instantiations of tweakable blockciphers and refinements to modes OCB and PMAC. In: Lee, P.J. (ed.) ASIACRYPT 2004. LNCS, vol. 3329, pp. 16-31. Springer, Heidelberg (2004)

52. Rogaway, P.: Formalizing human ignorance. In: Nguyên, P.Q. (ed.) VIETCRYPT 2006. LNCS, vol. 4341, pp. 211-228. Springer, Heidelberg (2006)

53. Shoup, V.: Lower bounds for discrete logarithms and related problems. In: Fumy, W. (ed.) EUROCRYPT 1997. LNCS, vol. 1233, pp. 256-266. Springer, Heidelberg (1997)

54. Zhang, W., Wu, W., Zhang, L., Feng, D.: Improved related-key impossible differential attacks on reduced-round AES-192. In: Biham, E., Youssef, A.M. (eds.) SAC 2006. LNCS, vol. 4356, pp. 15-27. Springer, Heidelberg (2007) 